\title{
Interferência Retroativa: O Esquecimento como uma Interrupção na Consolidação da Memória
}

\author{
Marcus Vinicius Costa Alves ${ }^{1}$ \\ Departamento de Psicobiologia da Universidade Federal de São Paulo, \\ São Paulo, SP, Brasil \\ Orlando Francisco Amodeo Bueno \\ Departamento de Psicobiologia da Universidade Federal de São Paulo, \\ São Paulo, SP, Brasil
}

\begin{abstract}
Resumo
O esquecimento é uma condição vivenciada diariamente pelos indivíduos e um conceito de extrema importância para a ciência da memória, apesar de sua experimentação ser complexa. Algumas teorias que tentam definir a ciência do esquecimento são apresentadas neste estudo, todavia, aqui focamos na Teoria da Interferência, principalmente na Interferência Retroativa (IR). A IR é a interferência que ocorre quando uma informação ou tarefa é inserida entre a apresentação de uma informação-alvo e sua posterior recordação. A IR pode ser explicada como uma competição de itens, mas atualmente surge a proposta que ela seja fruto da interrupção de um outro processo, chamado de Consolidação da Memória. A consolidação da memória é o processo através do qual as informações tornam-se estáveis, a partir de processos neurais posteriores ao registro inicial de uma informação que contribuem para o registro definitivo - ou, ao menos, mais duradouro - desta informação. A IR perturbaria estes processos posteriores à aprendizagem, resultando na perda destes materiais. O presente estudo visa propor a possibilidade da investigação mais aprofundada deste tópico para a melhor compreensão desse relevante conceito, visando aprofundar o conhecimento desta hipótese e outras possíveis causas do esquecimento.
\end{abstract}

Palavras-chave: Esquecimento, memória de longo prazo, Teoria da Interferência, consolidação da memória.

\section{Retroactive Interference: Forgetting as an Interruption of Memory Consolidation}

\begin{abstract}
El olvido es una condición experimentada diariamente por individuos y un concepto de suma importancia para la ciencia de la memoria. En el presente estudio algunas teorías que tratan de definir la ciencia de olvido serán discutidas y aquí nos centramos en la teoría de la interferencia, especialmente en la interferencia retroactiva (IR). El IR es la interferencia que se produce cuando se introduce una información o tarea entre la presentación de una información y su posterior recordación. Recientemente viene la propuesta de que la IR es el resultado de la interrupción de otro proceso, llamado consolidación de la memoria. Consolidación de la memoria es el proceso por el cual ocurre la estabilización de la infor-
\end{abstract}

Endereço para correspondência: Rua Botucatu, 862, Vila Clementino. São Paulo, SP, Brasil 04023062. Fone: (11) 21490155. E-mail: costaalves.mv@gmail.com

Os autores agradecem às instituições Fundação de Amparo à Pesquisa do Estado de São Paulo (FAPESP), Coordenação de Aperfeiçoamento de Pessoal do Nível Superior (CAPES) e Associação Fundo de Incentivo à Pesquisa (AFIP), que tornaram a realização deste estudo possível. 
mación, a partir de procesos neuronales posteriores al registro inicial de información que contribuyen al registro definitivo - o por lo menos, más duraderos - de esta información. El IR podría teóricamente perturbar estos procesos posteriores al aprendizaje, lo que resulta en la pérdida de estas informaciones. El presente estudio tiene como objetivo proponer una investigación mas profunda de este tema para comprender mejor este importante concepto, dirigido a profundizar el conocimiento de esta hipótesis y de otras posibles causas del olvido.

Keywords: Forgetting, long-term memory, interference theory, memory consolidation.

\section{Interferencia Retroactiva: Olvido como una Interrupción en la Consolidación de la Memoria}

\section{Resumen}

El olvido es una condición experimentada diariamente por individuos y un concepto de suma importancia para la ciencia de la memoria, a pesar de que el proceso de experimentación sea complejo. En el presente estudio algunas teorías que tratan de definir la ciencia de olvido serán discutidas, sin embargo, aquí nos centramos en la teoría de la interferencia, especialmente en la interferencia retroactiva (IR). El IR es la interferencia que se produce cuando se introduce una información o tarea entre la presentación de una información-objetivo y su posterior recordación. El IR se puede explicar como un elemento de competencia entre los elementos, pero recientemente viene la propuesta de que es el resultado de la interrupción de otro proceso, llamado consolidación de la memoria. Consolidación de la memoria es el proceso por el cual ocurre la estabilización de la información, a partir de procesos neuronales posteriores al registro inicial de información que contribuyen al registro definitivo - o por lo menos, más duraderos - de esta información. El IR podría teóricamente perturbar estos procesos posteriores al aprendizaje, lo que resulta en la pérdida de estas informaciones. El presente estudio tiene como objetivo proponer una investigación mas profunda de este tema para comprender mejor este importante concepto, dirigido a profundizar el conocimiento de esta hipótesis y de otras posibles causas del olvido.

Palabras clave: Olvido, memoria a longo plazo, Teoría de la Interferencia, consolidación.

Esquecer não é uma simples vis inertice [força inercial], mas uma força inibidora, ativa, graças à qual o que é por nós experimentado não penetra mais em nossa consciência ... não poderia haver felicidade, jovialidade, esperança, orgulho, presente, sem o esquecimento. (Nietzsche, 1887/1999)

O esquecimento é uma condição natural. Não conseguir recordar informações antes aprendidas é provavelmente uma das mais comuns aflições pelas quais as pessoas passam em seu dia a dia e, talvez por ser tão comum, tende a ser negligenciada como fator de imprescindível importância científica. Apesar de ser habitualmente rotulado como deletério e de causar certa exasperação, é possível também considerar o esquecimento como um fator adaptativo, sendo um processo necessário para a renovação de informações e a consequente adaptação do comporta- mento dos indivíduos ao ambiente, possibilitando também que eventualmente outras memórias possam ser registradas e selecionadas (Roediger, Weinstein, \& Agarwal, 2010; Storm, 2011). É possível considerar que o esquecimento também possui um papel autoprotetor, pois a impossibilidade de esquecer informações irrelevantes e inúteis poderia resultar em grande dificuldade de acessar informações específicas e valiosas de forma efetiva (Pergher \& Stein, 2003). Ademais, os raros relatos de indivíduos com uma capacidade de memória que os torna quase incapazes de esquecer revelam que esta condição pode ser tão exasperadora quanto a incapacidade de lembrar (Luria, 1968; Parker, Cahill, \& McGaugh, 2006; Storm, 2011).

Embora o esquecimento seja comumente experimentado pelas pessoas no dia a dia, cientificamente, até mesmo a comprovação da exis- 
tência de um fenômeno que possa ser chamado de "esquecimento" é um tópico complexo, demandando a atenção de psicólogos e neurocientistas. Afinal, para se estudar o esquecimento, a única medida que pode-se tomar é, a partir da incapacidade de se recordar informações antes aprendidas, inferir variáveis que engendrem tal condição. Apenas é possível especular se a informação antes passível de recordação foi-se completamente da memória que antes podia recuperá-la ou se a informação é impedida de ser recuperada por outros fatores (Davis, 2007).

Uma definição persistente de esquecimento é apresentada por Tulving (1974), segundo o qual este seria a inabilidade de recordar algo no presente, mas que podia ser recordado em um momento prévio. $\mathrm{O}$ esquecimento é um conceito de extrema importância para as ciências da memória, tendo em vista que permite a exposição de diversos processos mnemônicos, revelando como tipos de memórias se perdem diferentemente (McGeoch, 1932; Rubin, 2007).

A memória possui diversos sistemas, diferenciados a partir da observação do tipo de informação processada, o tempo de retenção desta informação e a capacidade de armazenamento desta informação (Squire, 2004). Sendo assim, se o processo cognitivo responsável pela retenção e recuperação de informações é complexo, a perda de informações neste processo também se torna multifacetado (Tulving, 1974).

\section{Teorias do Esquecimento}

Um dos pioneiros do estudo do esquecimento foi Ebbinghaus (Pergher \& Stein, 2003; Wixted, 2004). Analisando a retenção de informações (i.e.: listas de palavras) no decorrer do tempo, Ebbinghaus construiu sua famosa curva de esquecimento, na qual quanto maior tempo para a recuperação das informações, mais vertiginosa era a queda da quantidade de informações recordadas (Roediger et al., 2010).

Os experimentos de Ebbinghaus mostravam que uma informação perderia exponencialmente sua capacidade de ser revivida conforme o tempo - rapidamente no início e mais lentamente depois - sendo importantes para a formulação da hipóteses relacionadas a uma das teorias mais antigas sobre o esquecimento: a Teoria do Decaimento ou Deterioração (Decay Theory), teoria em que é sugerido que a perda de informação ocorre com o passar do tempo (Hardt, Nader, \& Nadel, 2013; Wixted, 2010).

A Teoria do Decaimento preconiza que as informações com o passar do tempo seriam aos poucos apagadas do sistema neural das pessoas, isto é, perdidas gradualmente de forma inexorável (Roediger et al., 2010).

Uma forma de teorizar sobre o esquecimento é considerá-lo uma perda absoluta das informações armazenadas (Davis, 2007). Sendo assim, o esquecimento seria o total desaparecimento dos traços de memória - informação armazenada e retida como resultado de uma percepção original de algum evento - de forma explícita ou implícita, não havendo possibilidade de recordá-las, não importando quais técnicas sejam utilizadas para tal tentativa de recordação (Davis, 2007; Tulving, 1974). Embora exista a dificuldade de comprovar a inexistência dos traços de memória perdidos, a possibilidade explicativa advinda desta teoria se fortalece com a ideia de que diversos aspectos da vida dos indivíduos durante o seu desenvolvimento não podem ser recordados, independentemente das técnicas e pistas utilizadas para tentar trazer tais memórias à tona (Roediger et al., 2010).

A perda das informações com o passar do tempo, apesar de plausível, não pode ser considerada a única forma de esquecimento, em primeiro plano porque explicar o esquecimento desta forma seria afirmar que as memórias seriam como músculos - se atrofiando quando não usadas - se abstendo de especificar um mecanismo que cause esse esquecimento, e por outro, esta teoria não consegue justificar estudos que revelam que uma série de fatores diversos - que não apenas o tempo - podem intervir na fixação das memórias (Lechner, Squire, \& Byrne, 1999; Roediger et al., 2010; Wixted, 2004).

Outra teoria para o esquecimento, a Teoria da Recuperação, é a de que este é proveniente da incapacidade de recuperar informações, ou seja, as informações, ainda que estejam presentes no cérebro dos indivíduos, não estariam aptas para a recuperação (Roediger et al., 2010; Wixted, 
2010). O esquecimento seria um fenômeno dependente de pistas, ou seja, refletiria a falha da recuperação de um traço de memória perfeitamente intacto, devido a mudanças no contexto cognitivo dos indivíduos (Tulving, 1974). Basicamente, a teoria defende que não necessariamente o traço da memória esteja perdido para sempre quando há uma impossibilidade de sua recordação, mas sim que ele pode apenas estar inacessível (Tulving, 1974). Comumente, tarefas de reconhecimento a partir de pistas são utilizadas para a testagem desta assertiva, tendo em vista que as pistas ativam o traço de memória já registrado acerca de uma informação, facilitando a recuperação desta (Tulving, 1974; Wixted, 2010).

Outra teoria proeminente para o estudo do esquecimento e da perda deinformações éa Teoria da Interferência, que afirma que o esquecimento é causado por informações intervenientes que interferem na informação-alvo (Lechner et al., 1999; Wixted, 2004). A Teoria da Interferência é uma das teorias clássicas mais importantes para a compreensão do esquecimento (McGeoch, 1932; Pergher \& Stein, 2003; Wixted, 2004).
Por muitos anos esta teoria era o paradigma mais utilizado para explicar o esquecimento, e embora tenha passado por diversas críticas durante os anos que se seguiram à sua proposição - chegando inclusive a ser deixada de lado por algumas décadas - atualmente está ressurgindo como uma das explicações mais eficientes para o esquecimento, a partir da proposta de que processos supervenientes à aprendizagem podem influenciar a perda de informações pelos indivíduos (Dewar, Cowan, \& Della Sala, 2007; Wixted, 2004).

A Teoria da Interferência consiste na proposição de que informações disputam entre si, sobrepondo-se nos sistemas de memórias (Dudai, 2004; Wixted, 2004). A competição entre informações pode se referir a itens aprendidos anteriormente (Interferência Proativa, IP) ou posteriormente (Interferência Retroativa, IR). Os paradigmas clássicos para experimentos com interferência são: ao se apresentar um esquema A-B de recordação, a primeira informação (A) e a segunda (B) disputam sua existência, quando A interfere com B, a interferência é Proativa; e quando $\mathrm{B}$ interfere com $\mathrm{A}$, a interferência é Retroativa (Figura 1).

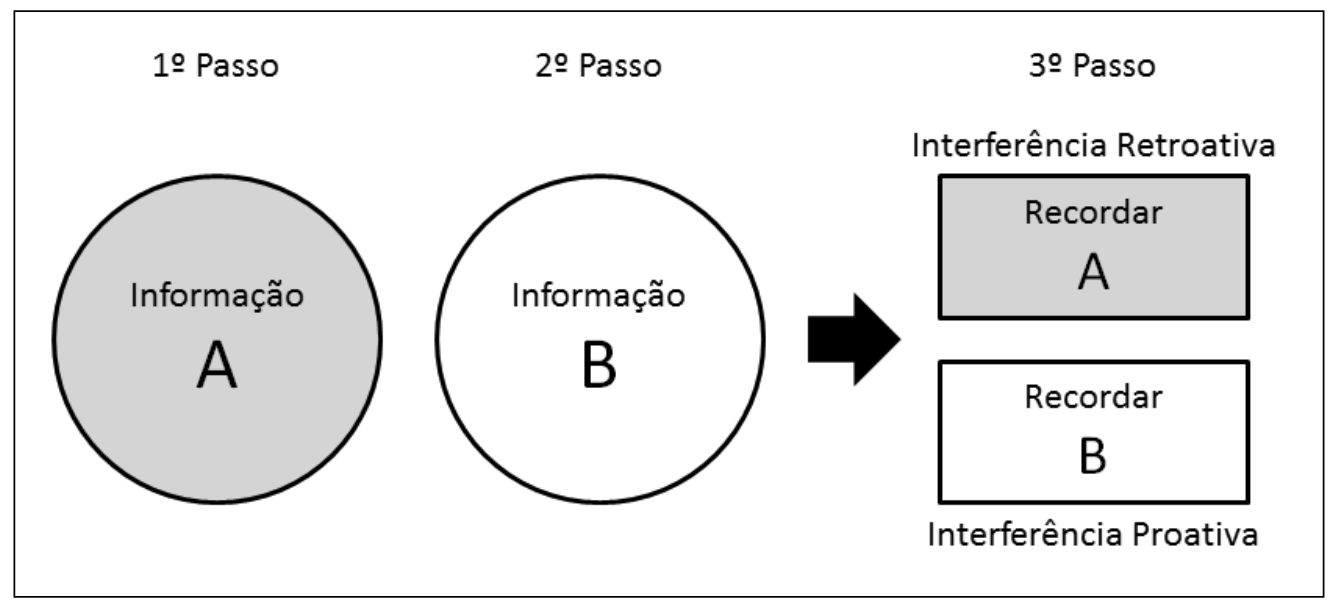

Figura 1. Paradigmas clássicos da Teoria da Interferência. Imagem retirada de Alves (2013).

Em certo momento houve uma redução de investigações que buscavam explicar o esquecimento apenas com o foco nesta teoria, principalmente com o foco na IR (Anderson, 2003). Um dos responsáveis por esta mudança na forma de observar o esquecimento foi o trabalho crítico de
Underwood em 1957, que sugeriu que a maioria da informação perdida se deveria à IP e não à IR. O trabalho deste autor, por sua influência, fez que por anos houvesse um hiato nas investigações das influências da IR no esquecimento e na consolidação das memórias. 
Todavia, a crítica feita pelo estudo de Underwood (1957) com o passar do tempo esmoreceu e estudos recentes sugerem que a IR é um fator consistente para o esquecimento (Cowan, Beschin, \& Della Sala, 2004; Dewar, Cowan, \& Della Sala, 2007, 2010; Lechner et al., 1999; Wixted, 2004, 2010). A utilização da IR em experimentos voltou a ser considerada, sem que o interesse pela IP fosse negligenciado (Wixted \& Rohrer, 1993). Sendo assim, entender como as interferências posteriores à aprendizagem influenciam a perda de informações ainda é um tópico aberto para amplas investigações na ciência da memória (Wixted, 2004).

\section{O Retorno da Interferência Retroativa como Possível Explicação para o Esquecimento}

A IR surge com os trabalhos de Müller e Pilzecker em 1900 (apud Lechner et al., 1999), inicialmente chamada de Inibição Retroativa (Anderson, 2003). A IR foi inicialmente definida como a interferência que ocorre quando uma informação ou tarefa é inserida entre a apresentação de uma informação-alvo e a posterior recordação desta mesma informação (Cowan et al., 2004; Dewar et al., 2007; Lechner et al., 1999; McGaugh, 1999).

A Teoria da Interferência pode ser facilmente explicada como um caso de Teoria da Recuperação: as tarefas interferentes, proativas ou retroativas, diminuiriam a discriminabilidade dos itens a serem recuperados, gerando uma confusão entre eles. Uma série de pesquisadores tendem a interpretar o efeito da Interferência na recuperação de informações como derivada de um processo de inibição (Anderson, 2003; Anderson, Green, \& McCulloch, 2000; Anderson \& Spellman, 1995; Fawcett \& Taylor, 2008). Para Anderson (2003), o esquecimento aconteceria pelos mecanismos de controle envolvidos na inibição de informações irrelevantes, ou seja, a interferência seria um processo advindo de sistemas de controle mental e comportamental. Consequentemente, o esquecimento aconteceria não por um processo passivo de perda, mas sim por um processo inibitório em que cognitivamente algumas informações seriam mais importantes que outras para uma futura recuperação, e com isso, as informações menos relevantes seriam suprimidas por estas (Anderson, 2003; Fawcett \& Taylor, 2008). Segundo esta perspectiva, o ato de lembrar algumas informações - e assim, tornando-as mais relevantes - consequentemente faz com que outras sejam perdidas (Anderson, 2003; Anderson, Bjork, \& Bjork, 1994; Chan, Erdman, \& Davis, 2015). Esta ideia traz diversos desafios metodológicos e com consequências teóricas muito interessantes. Segundo essa proposta, a mera visão de que estímulos e traços interferentes causam o esquecimento é pouco funcional, trazendo então a proposta de que, seja intencional ou incidental, o esquecimento seria fruto de uma resposta à interferência causada pela ativação de competidores na memória.

Um estudo recente realizado com crianças e adolescentes apresentou evidências de que crianças sofrem um efeito ostensivo da IR (Darby \& Sloutsky, 2015). O estudo de Darby e Sloustky (2015) demonstrou que independente da faixa etária dos participantes, o efeito da IP não se apresentava diferente. Já em relação à IR, as entradas de estímulos interferentes se mostrou, de acordo com os autores, catastrófico, mas apenas para as crianças. Esse resultado corrobora com a hipótese de que processos executivos estão relacionados à IR, tendo em vista que crianças com desenvolvimento executivo menor seriam mais propensas a erros inibitórios, e portanto, estariam mais suscetíveis à interferência.

Este seria outro fator que indicaria a possibilidade de competição na Recuperação ser o fator mais importante na IR, pois a competição dos estímulos intervenientes seria também dependente dos recursos atencionais dos indivíduos, com o uso de recursos para separar o que guardar e o que não guardar quando todas as informações são previamente passíveis de recordação. E assim, com a maturação do sistema cognitivo, as pessoas se tornariam menos suscetíveis ao efeito da IR (Lewis-Peacock \& Norman, 2014).

Críticas à Teoria da Interferência Retroativa. Apesar de ser uma das teorias que retomou sua posição de valor científico mais forte e recentemente vem sendo observada com grande interesse, é preciso lembrar que argumentos 
poderosos contra o olhar generalista das interferências foram levantados há algum tempo. Em sua revisão, já citada neste estudo, Underwood (1957) abordava justamente a característica generalista do argumento proposto na Teoria da Interferência. A clássica revisão de Underwood aponta não para a inexistência da interferência como explicação do esquecimento, mas principalmente para o uso desmedido de justificativas segundo essa teoria para explicar, por exemplo, o decréscimo de recordação em indivíduos após $24 \mathrm{~h}$ de acontecido o experimento. Diversos estudos relatavam resultados diferentes para a recordação após esse período e todos imputavam tal perda à diferentes IR em que as pessoas estariam suscetíveis durante o dia, mas sem uma explicação diligente. Segundo uma explicação generalista, as pessoas provavelmente perderiam as informações adquiridas durante o experimento pela competição com as informações diárias adquiridas pós-laboratório.

O que Underwood (1957) demonstrou foi que esse efeito vinha principalmente da quantidade de informação prévia - listas de palavras - apresentada ao indivíduo, o tornando suscetível a não fixação de uma grande quantidade de informação durante o experimento, ou seja, para ele, a perda de informações pelos indivíduos estaria relacionada à IP, relegando à IR uma pequena parte do esquecimento. Outrossim, neste mesmo trabalho, há o argumento de que o efeito interferente principal se daria principalmente pela similaridade dos itens e não devido à competição de informações do dia do indivíduo.

O problema trazido por esta proposta vem com os dados encontrados em experimentos de IR e sono: tendo em vista que diversos estudos mostram que um período de sono após adquirir alguma informação tende a evitar sua perda (Ekstrand, 1967; Jenkins \& Dallenbach, 1927), ou seja, o sono reduziria a IR, evitando a competição de informações.

Apesar dos estudos que indicam influência atencional e executiva no esquecimento proveniente da Teoria da Interferência, outra explicação que se encontra em voga na atualidade é proveniente da Teoria da Consolidação da Memória.

\section{Interferência Retroativa na Consolidação} da Memória. A existência da IR pode também ser explicada através de um processo hipotético sugerido também pela primeira vez no trabalho seminal de Müller e Pilzecker em 1900, chamado de Consolidação da Memória (Lechner et al., 1999; McGaugh, 1999, 2000). A Teoria da Consolidação descarta a Teoria da Recuperação como o fator preponderante a causar o esquecimento, pois segundo esta teoria, não seria pela competição de informações relevantes durante a recuperação que a memória se perderia.

A consolidação da memória é o processo através do qual as informações tornam-se estáveis com o passar do tempo (Dudai, 2012; Nadel \& Bohbot, 2001). A concepção que baliza a consolidação da memória é a de que processos neurais posteriores ao registro inicial de uma informação contribuem para o registro mais duradouro desta informação, fortalecendo os traços da memória (McGaugh, 2000; Nadel \& Bohbot, 2001; Nadel \& Moscovitch, 1997). A consolidação refere-se assim, à progressiva estabilização de alguma informação após a sua aquisição, e sendo assim, uma nova memória necessitaria de tempo para se estabilizar (Dudai, 2004, 2012).

O processo de Consolidação da Memória tende a ser compreendido em dois níveis, um celular/sináptico e o outro de sistemas. No nível sináptico, a consolidação seria a estabilização de uma informação após sua codificação em uma memória de longo-prazo em nodos sinápticos e celulares específicos no circuito neural que codificam a memória, podendo ser considerada uma parte da consolidação sistêmica (Dudai, 2012; Dudai, Karni, \& Born, 2015; Izquierdo \& Medina, 1997). Na presente revisão, focaremos na Consolidação da Memória em sistemas, ou seja, a reorganização de informações após a sua codificação em representações distribuídas em circuitos cerebrais de memória de longo-prazo (Dudai et al., 2015).

Durante a consolidação, em que processos críticos para a fixação das memórias acontecem, estas estariam suscetíveis a agentes amnésicos, tais como informações interferentes, tarefas que demandem esforço cognitivo, choques eletroconvulsivos, toxinas, determinadas drogas, 
lesões cerebrais, estimulação magnética transcraniana etc. A IR perturbaria estes processos posteriores à aprendizagem, resultando na perda destes materiais (Dewar, Cowan, et al., 2010; Robertson, 2012). Logo, a IR aconteceria não só com a aprendizagem de novas memórias concorrentes, mas também com qualquer estímulo posterior à aprendizagem que possua a capacidade de interromper ou desregular o processo de consolidação (Figura 2; Dewar et al., 2007; Dudai, 2004; McGaugh, 1966, 2000; Robertson, 2012; Squire \& Alvarez, 1995). No entanto, após algum tempo, tais situações perturbadoras perderiam sua capacidade de influência negativa sobre a memória. A memória em questão pode- ria enfim ser considerada consolidada, ou seja, pouco susceptível a disrupções (Dudai, 2004; McGaugh, 1966; Wixted, 2004). A consolidação definitiva de uma memória poderia indicar que esta informação estaria agora impassível a qualquer fator que pudesse previamente a perturbar, contudo esta visão tem sido desafiada com trabalhos recentes que sugerem que a reativação de informações outrora consolidadas tendem a fazer com que estas memórias se tornem lábeis, ou seja, novamente suscetíveis à mudanças e sujeita a transformações e adaptações, um processo denominado Reconsolidação da Memória (Dudai, 2004, 2012; Dudai et al., 2015; Gisquet-Verrier et al., 2015).

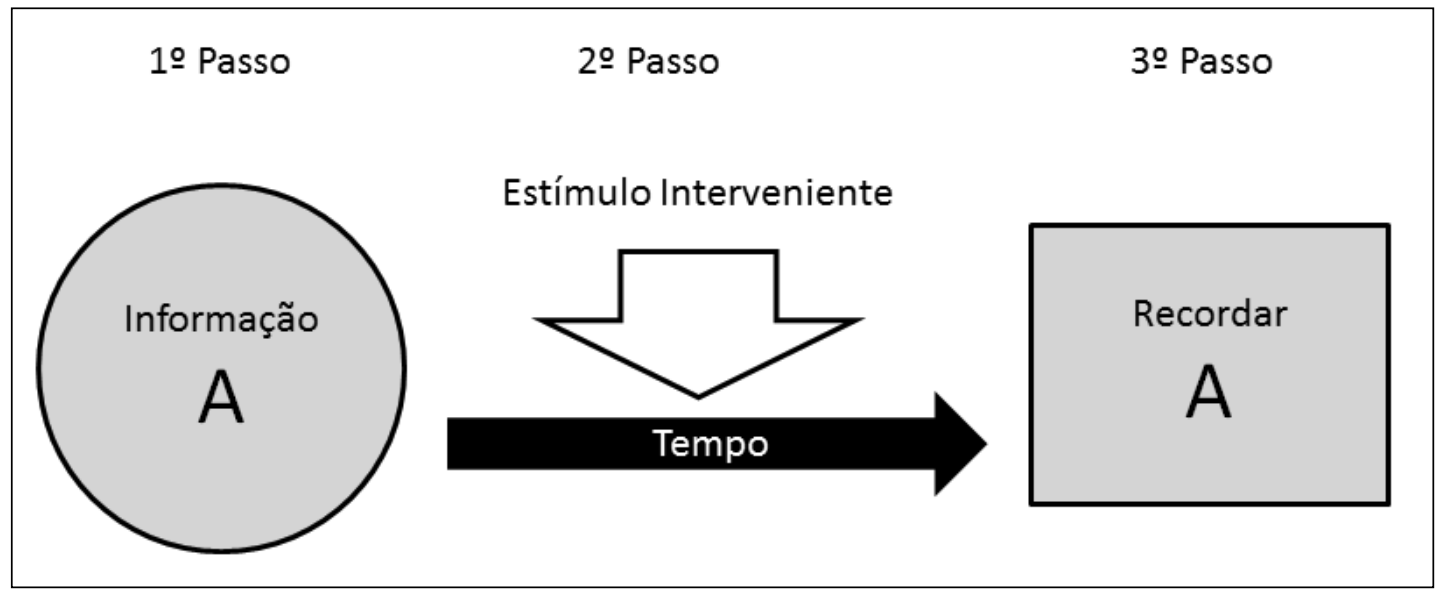

Figura 2. Interferência Retroativa durante a consolidação da memória. Imagem retirada de Alves (2013).

O tempo necessário para que a consolidação seja afetada não é matéria consensual entre cientistas, tendo em vista evidências de que a consolidação pode transcorrer por um tempo muito variável e que os estágios posteriores, nos quais ocorrem a reorganização dos processos neurais, podem durar de alguns minutos até alguns anos (Dudai, 2004; Lechner et al., 1999; McGaugh, 2000). Ressalta-se que a Teoria da Consolidação reflete a ideia de perda irrecuperável de informações, pois aquelas não consolidadas não podem ser recuperadas. É visível que a noção do conceito de Consolidação da Memória se parece com o conceito de IR, tendo em vista que desde os trabalhos seminais neste tópico as descobertas fundamentais acerca dos processos de consolidação mostram que interrupções interferem neste processo (McGaugh, 2000). O que muda é a ideia de que os agentes interferentes não são intrinsicamente relacionados ao material a ser lembrado.

Estruturalmente, o lobo temporal medial, ou, mais especificamente, a formação hipocampal - comumente referida como consistindo de hipocampo, giro denteado, subículo e córtex entorrinal (Wixted, 2004) - parece ter uma grande relação com a consolidação da memória, considerando que se esta formação sofrer algum dano antes do processo de consolidação estar completo, memórias ainda não consolidadas não serão passíveis de recordação (Lechner et al., 1999; Nadel \& Moscovitch, 1997; Wixted, 2004). O registro definitivo das memórias se daria pelo gradual armazenamento destas - antes depen- 
dentes do sistema hipocampal - no neocórtex, a partir da reorganização da circuitaria cerebral após a codificação da informação pela constante perseveração de uma informação aprendida, que se torna independente do hipocampo (Dudai, 2012; Lechner et al., 1999; McGaugh, 1999; Squire \& Alvarez, 1995; Wixted, 2010). Apesar de normalmente pesquisadores atribuírem a mecanismos inibitórios da cognição - relacionados ao Córtex Pré-Frontal - a existência da IR (Anderson, 2003; Anderson et al., 2000; Anderson \& Spellman, 1995), há também a hipótese de que a formação hipocampal - importante para a consolidação - também esteja relacionada com a perda de informação na IR, pois o hipocampo seria fundamental para reduzir a suscetibilidade à interferência segundo a interpretação de alguns autores (Andrejkovicks, Balla, \& Bereczki, 2013; Winocur, Becker, Luu, Rosenzweig, \& Wojtowicz, 2012; Wixted, 2004).

Para a compreensão desta progressiva estabilização das informações, estudos com pacientes amnésicos parecem ser um bom indicativo da existência do processo de consolidação e das estruturas que permeiam tal processo (Cowan et al., 2004; Dewar et al., 2007; Dudai, 2004, 2012). Pacientes que sofreram lesões no lobo temporal medial e apresentam Amnésia Anterógrada - a incapacidade de reter novos eventos, apesar da existência da manutenção de novas memórias por um curto espaço de tempo - podem apresentar também uma leve Amnésia Retrógrada, ou seja, a perda de certa quantidade de informação pré-evento causador da amnésia (Squire, \& Alvarez, 1995). Essa pequena perda pode indicar exatamente que estes materiais ainda não haviam sido consolidados (Dudai, 2012; Lechner et al., 1999; Squire \& Alvarez, 1995). Entretanto, o que caracteriza melhor o efeito de lesões no lobo temporal medial é a Amnésia Anterógrada de Memórias de Longo-Prazo, que sugere que processos posteriores ao recebimento da informação são necessários para que ocorra a fixação destas memórias no cérebro, processo que nestes pacientes se tornaram inviáveis devido ao fator causador da amnésia (Dewar, Della Sala, Beschin, \& Cowan, 2010; Nadel \& Bohbot, 2001; Nadel \& Moscovitch, 1997).
Recentes estudos com listas de palavras com pacientes com amnésia anterógrada revelaram que, quando uma informação é apresentada para a posterior recordação é seguida de um intervalo onde não há realização de tarefa nenhuma, ocorre uma maior recordação da informação-alvo (Cowan et al., 2004; Dewar, Della Sala, et al., 2010). Argumenta-se que este efeito exista devido às características da consolidação da memória. Uma particularidade desse tipo de condição seria a de que pacientes com déficits relacionados à amnésia possuem menores recursos para a consolidação, o que faz com que os efeitos de degradação dos traços de memória provenientes da IR sejam consideravelmente mais visíveis (Brown, 2002; Cowan et al., 2004; Dewar, Cowan, et al., 2010). Além disso, tais resultados seriam uma indicação de que um intervalo onde nenhuma atividade cognitiva é empenhada possibilitaria a ocorrência de processos neurais necessários para a consolidação. Assim, o esforço cognitivo empenhado em tarefas posteriores à aprendizagem surge como um possível fator de considerável influência na consolidação da memória (Cowan et al., 2004; Dewar, Cowan, et al., 2010).

Os efeitos que podem conduzir a um aumento ou diminuição da IR são variáveis; muito da informação perdida pelos indivíduos se deve a condições não específicas provenientes da IR, como o tipo de tarefa realizada nessa interferência, o contexto na realização dos testes ou o tipo de codificação no sistema de memória requerido para a realização das tarefas e para a recordação da informação-alvo (Robertson, 2009, 2012; Unsworth, Brewer, \& Spiller, 2013; Wixted, 2004), e embora a gama de possibilidades de interferência seja extensa (similaridade, esforço cognitivo, agentes amnésicos, drogas etc.), aparentemente os resultados obtidos nos estudos de IR parecem ser bem parecidos e podem ser interpretados como consequências de estímulos intervenientes que afetam o processo de consolidação. Duas questões devem ser levantadas: quanto do traço da memória já estava consolidado quando o fator de interferência surgiu e como este fator afeta os processos neurais subsequentes que são fundamentais para esta consolida- 
ção (Dewar et al., 2007; Dewar, Garcia, Cowan, \& Della Sala, 2009; Robertson, 2012; Wixted, 2004).

$\mathrm{O}$ argumento em favor da ideia de que ocorre uma IR gerando o esquecimento se torna ainda mais plausível ao se considerar diversos trabalhos em que seus autores sugerem que quando a aquisição da informação é seguida de um intervalo sem realizar qualquer tarefa, as pessoas conseguem recordar informações com mais facilidade (Abel \& Bäuml, 2012; Dewar et al., 2007; Dewar, Della Sala, et al., 2010; Wixted, 2010). Logo, o paradigma mais utilizado para se contrapor a IR consiste em pospor um intervalo onde os participantes não realizam tarefa alguma e são instruídos a não realizar reverberação (vocal ou subvocal) da informação previamente apresentada (Dewar et al., 2007; Wixted, 2004).

Críticas à Teoria da Consolidação da Memória. Uma das contestações sobre a teoria da Consolidação da Memória é, por exemplo, a de que Muller e Pilzecker teorizaram que a consolidação duraria por pequenos períodos, menos de dez minutos (Lechner et al., 1999), mas tal fato não é encontrado em estudos humanos, tendo em vista que a consolidação pode levar de alguns minutos até algumas horas ou mais (Brown, 2002). O tempo necessário para que a consolidação de uma memória ocorra é ainda um tópico de extremo debate, enquanto podemos compreender que algumas informações são lábeis por um grande período - podendo ser danificadas - é também claro para a psicologia cognitiva que é possível reter informações quase que imediatamente em curto-prazo (McGaugh, 2000). Uma explicação possível para essa questão é a de que o processo de Consolidação da Memória não é apenas pontual, mas ocorre desde o início da aquisição da memória e a sua lenta duração advém de uma função adaptativa que permite que processos endógenos ativados pela experiência modulem a força da memória (McGaugh, 2000), e por isso algumas memórias se tornariam mais estáveis que outras.

A consolidação é pouco utilizada para explicações teóricas entre psicólogos e neurocientistas que trabalham com seres humanos devido à dificuldade de testa-la de forma diligente em humanos, enquanto que em animais a teoria se encontra extremamente bem estabelecida (McGaugh, 2000). Os dados que remetem ao estudo e aos modelos do esquecimento têm tomado uma forma diferente em estudos com viés comportamental, quase não se aborda o termo "Consolidação da Memória", enquanto estudos em não-humanos abordam a consolidação extensivamente. Partindo desse ponto, há a possibilidade de se falar de esquecimento sem precisar remeter ao processo de consolidação da memória, e, com isso, abordar meramente o conteúdo comportamental deste processo. Todavia, apesar das críticas, a Teoria da Consolidação é importante para explicar os processos posteriores à aquisição de uma informação, relembrando que estudos em animais não-humanos já têm demonstrado com eficiência que este processo ocorre.

\section{Conclusão}

Os estudos recentes demonstram a possibilidade do retorno da Teoria da Interferência como proposta plausível para o esquecimento, ademais, relacionando esta teoria com a da Consolidação da Memória, o que torna possível adentrar o escopo investigativo que juntaria estudos em humanos e não-humanos, alcançando então uma relação mais proeminente para a investigação científica. Apesar de existirem críticas às duas teorias, o modelo tem sido utilizado com afinco nos últimos anos para a explicação do fenômeno do esquecimento em uma tentativa de conciliar investigações em animais e humanos saudáveis ou com algum prejuízo cognitivo. Tendo em vista que a consolidação seria um decréscimo na fragilidade dos traços de memória, teorias e estudos em relação à memória devem abordar a consolidação como um fenômeno influente neste processo (Brown, 2002). Do ponto de vista neurofisiológico o esquecimento ao longo do tempo mobiliza mecanismos fisiológicos que mediariam o esquecimento tempo-dependente (Dong et al., 2016; Sachser et al., 2016; Villareal, Do, Haddad, \& Derrick, 2002). Segundo estes achados recentes, a memória poderia durar indefinidamente, contanto que seja protegida do esquecimento ativo associado a mecanismos 
neuroquímicos específicos. Esta possibilidade abre uma perspectiva completamente nova e inesperada de tratamento para disfunções patológicas da memória.

O presente estudo propõe então que mais estudos experimentais sejam realizados para o melhor escrutínio deste tópico, realizados rigorosamente em prol da investigação das causas fundamentais do esquecimento. Além disso, são necessários mais estudos clínicos, com pacientes com amnésia ou que possuam algum déficit de memória que os tornem mais suscetíveis a múltiplas interferências, permitindo analisar como esse fator se relaciona com voluntários saudáveis. Ademais, é importante também que os estudos vão além das formas clássicas de investigação em Interferência, indo além dos clássicos pares de palavras, evidenciando a investigação de outras possíveis variáveis intervenientes, como o esforço cognitivo implicado em tarefas interferentes, demandas atencionais e executivas, demandas de memória em curto-prazo e o tempo levado para que tarefas se tornem mais ou menos interferentes.

O estudo do esquecimento é importante para a ciência da memória, pois este é um tópico que permite levantar questões e encontrar respostas frutíferas para o entendimento da memória em si. A capacidade de lembrar é fundamental para a sobrevivência, afinal, lembrar de algo permite prever o que acontecerá no futuro e adaptar o comportamento a esses acontecimentos. Já o esquecimento, é um conceito que apesar de estar presente diariamente na vida das pessoas e de ser amplamente vivenciado, não possui ainda uma definição científica e metodológica consensual, todavia, sua existência também é fruto de questões adaptativas, afinal, esquecer o que é irrelevante nos permite lembrar o que é essencial.

\section{Referências}

Abel, M., \& Bäulm, K. H. (2012). Sleep can eliminate list-method directed forgetting. Journal of Experimental Psychology: Learning, Memory, and Cognition, 22, 1-7. doi:10.1037/a0030529

Alves, M. V. C. (2013). Interferência retroativa de diferente demandas cognitivas na consolidação da memória (Dissertação de mestrado em Ciên- cias, Departamento de Psicobiologia, Universidade Federal de São Paulo, Escola Paulista de Medicina, SP, Brasil)

Anderson, M. C. (2003). Rethinking interference theory: Executive control and the mechanisms of forgetting. Journal of Memory and Language, 49, 415-445. doi:10.1016/j.jml.2003.08.006

Anderson, M. C., Bjork, R. A., \& Bjork, E. L. (1994). Remember can cause forgetting: Retrieval dynamics in Long-Term Memory. Journal of Experimental Psychology: Learning, Memory and Cognition, 20(5), 1063-1087.

Anderson, M. C., Green, C., \& McCulloch, K. C. (2000). Similarity and inhibition in Long-Term Memory: Evidence for a Two-Factor Theory. Journal of Experimental Psychology: Learning, Memory and Cognition, 26(5), 1141-1159. doi:10.1037M1278-7393.26.5.I141

Anderson, M. C., \& Spellman, B. A. (1995). On the status of inhibitory mechanisms in cognition: Memory retrieval as a model case. Psychological Review, 102(1), 68-100.

Andrejkovicks, M., Balla, P., \& Bereczki, D. (2013). Susceptibility to interference and intrusion errors in consequence of the dominant hemisphere's hipocampal infarct: A case report. Neurocase, 20(2). doi:10.1080/13554794.2012.741256

Brown, A. (2002). Consolidation theory and retrograde amnesia in humans. Psychonomic Bulletin \& Review, 9(3), 403-425. doi:http://dx.doi. org/10.3758/BF03196300

Chan, J. C. K., Erdman, M. R., \& Davis, S. D. (2015). Retrieval induces forgetting, but only when nontested items compete for retrieval: Implication for interference, inhibition, and context reinstatement. Journal of Experimental Psychology: Learning, Memory, and Cognition. doi:http:// dx.doi.org/10.1037/xlm0000101

Cowan, N., Beschin, N., \& Della Sala, S. (2004). Verbal recall in amnesiacs under conditions of diminished retroactive interference. Brain, 127, 825-834. doi:10.1093/brain/awh107

Darby, K. P., \& Sloutsky, V. M. (2015). The cost of learning: Interference effects in memory development. Journal of Experimental Psychology: General, 144(2), 410-431. doi:http://dx.doi. org/10.1037/xge0000051

Davis, M. (2007). Forgetting: Once again, it's all about representations. In H. L. Roediger III, Y. Dudai, \& S. M. Fitzpatrick (Eds.), Science of 
Memory (pp. 317-319). New York: Oxford University Press.

Dewar, M. T., Cowan, N., \& Della Sala, S. (2007). Forgetting due to retroactive interference: A fusion of early insights into everyday forgetting and recent research on anterograde amnesia from Müller and Pilzecker's (1900). Cortex, 43(5), 616-634.

Dewar, M. T., Cowan, N., \& Della Sala, S. (2010). Forgetting due to retroactive interference in amnesia: Findings and implications. In S. Della Sala (Ed.), Forgetting (pp. 185-209). New York: Psychology Press.

Dewar, M. T., Della Sala, S., Beschin, N., \& Cowan, N. (2010). Profound retroactive interference in anterograde amnesia: What Interferes? Neuropsychology, 24(3), 357-367. doi:10.1037/ a0018207

Dewar, M. T., Garcia, Y. F., Cowan, N., \& Della Sala, S. (2009). Delaying interference enhances memory consolidation in amnesic patients. Neuropsychology, 23(5), 627-634. doi:10.1037/ a0015568

Dong, Z., Han, H., Li, H., Bai, Y., Wang, W., Tu, M., Peng, Y., ...Wang, Y. T. (2016). Long-term potentiation decay and memory loss are mediated by AMPAR endocytosis. Journal of Clinical Investigation, 125(1), 234-247. doi:10.1172/ JCI77888

Dudai, Y. (2004). The Neurobiology of Consolidation, or, how stable is the engram? Annual Review of Psychology, 55, 51-86. doi:10.1146/annurev.psych.55.090902.142050

Dudai, Y. (2012). The Restless Engram: Consolidation never end. Annual Review of Neuroscience, 227-247. doi:10.1146/annurev-neuro-062111-150500

Dudai, Y., Karni, A., \& Born, J. (2015). The consolidation and transformation of memory. Neuron, 88(1), 20-32. doi:http://dx.doi.org/10.1016/j. neuron.2015.09.004

Ekstrand, B. R. (1967). Effect of sleep on memory. Journal of Experimental Psychology, 75(1), 6472. doi:http://dx.doi.org/10.1037/h0024907

Fawcett, J. M., \& Taylor, T. L. (2008). Forgetting is effortful: Evidence from reaction time probes in an item-method directed forgetting task. Memory \& Cognition, 36(6), 1168-1181. doi:10.3758/ MC.36.6.1168
Gisquet-Verrier, P., Lynch, L. F., III, Cutolo, P., Toledano, D., Ulmen, A., Jasnow, A. M., \& Riccio, D. C. (2015). Integration of new information with active memory accounts for retrograde amnesia: A challenge to the consolidation/reconsolidation hypothesis? The Journal of Neuroscience, 35(33), 11623-11633. doi:10.1523/JNEUROSCI.1386-15.2015

Hardt, O., Nader, K., \& Nadel, L. (2013). Decay happens: The role of active forgetting in memory. Trends in Cognitive Sciences, 17(3), 111-120. doi:http://dx.doi.org/10.1016/j.tics.2013.01.001

Jenkins, J. G., \& Dallenbach, K. M. (1927). The effect of serial position upon recall. The American Journal of Psychology, 38, 285-291. doi:http:// dx.doi.org/10.2307/1415207

Izquierdo, I., \& Medina, J. H. (1997). Memory formation: The sequence of biochemical events in the hippocampus and its connection to activity in other brain structures. Neurobiology of Learning and Memory, 68, 285-316.

Lechner, H. A., Squire, L. R., \& Byrne, J. A. (1999). 100 Years of Consolidation-- Remembering Müller and Pilzecker. Learning \& Memory, 6, 77-87. doi:10.1101/lm.6.2.77

Lewis-Peacock, J. A., \& Norman, K. A. (2014). Competition between items in working memory leads to forgetting. Nature Communications, 5, 1-10. doi:10.1038/ncomms6768

Luria, A. R. (1968). The Mind of a Mnemonist: $A$ little book about a Vast Mind. New York: Basic Books.

McGaugh, J. L. (1966). Time-dependent process in memory storage. Science, 153, 1351-1358.

McGaugh, J. L. (1999). The perseveration-consolidation hyphothesis: Mueller and Pilzecker, 1900. Brain Research Bulletin, 50, 445-446.

McGaugh, J. L. (2000). Memory: A Century of Consolidation. Science, 287, 248-251. doi: $10.1126 /$ science. 287.5451 .248

McGeoch, J. A. (1932). Forgetting and the law of disuse. Psychological Review, 39, 352-370. doi:http://dx.doi.org/10.1037/h0069819

Nadel, L., \& Bohbot, V. (2001). Consolidation of memory. Hippocampus, 11, 56-60. doi:10.1002/1098-1063(2001)11:1<56::AID-HIPO1020>3.0.CO,2-O 
Nadel, L., \& Moscovitch, M. (1997). Memory consolidation, retrograde amnesia and the hippocampal complex. Current Opinion in Neurobiology, 7,217-227.

Nietzsche, F. (1999). Genealogia da moral (P. C. de Souza, Trans.). São Paulo, SP: Companhia das Letras. (Original publicado em 1887)

Parker, E. S., Cahill, L., \& McGaugh, J. L. (2006). A Case of Unusual Autobiographical Remembering. Neurocase: The Neural Basis of Cognition, 12(1), 35-49. doi:http://dx.doi. org/10.1080/13554790500473680

Pergher, G. K., \& Stein, L. M. (2003). Compreendendo o esquecimento: Teorias clássicas e seus fundamentos experimentais. Psicologia USP, 14(1), 129-155. doi:10.1590/S010365642003000100008

Robertson, E. M. (2009). From creation to consolidation: A novel framework for memory processing. PLoS Biology, 7(1), 11-19. doi:10.1371/ journal.pbio.1000019

Robertson, E. M. (2012). New insights of human memory, interference and consolidation. Current Biology, 22, R66-R71. doi 10.1016/j. cub.2011.11.051

Roediger, H. L., III, Weinstein, Y., \& Agarwal, P. K. (2010). Forgetting: Preliminary considerations. In S. Della Sala (Ed.), Forgetting (pp. 1-22). New York: Psychology Press.

Rubin, D. C. (2007). Forgetting: Its role in the Science of memory. In H. L. Roediger III, Y. Dudai, \& S. M. Fitzpatrick (Eds.), Science of Memory (pp. 325-328). New York: Oxford University Press.

Sachser, R. M., Santana, F., Crestani, A. P., Lunardi, P., Pedraza, L. K., Quillfeldt, J. A., Hardt, O., \& Alvares, L. O. (2016). Forgetting of long-term memory requires activation of NMDA receptors, L-type voltage-dependent $\mathrm{Ca} 2+$ channels, and calcineurin. Scientific Reports, 6, 22771. doi:10.1038/srep22771

Squire, L. R. (2004). Memory system of the brain: A brief history and current perspective. Neurobiology of Learning and Memory, 82, 171-177. doi:10.1016/j.nlm.2004.06.005

Squire, L. R., \& Alvarez, P. (1995). Retrograde amnesia and memory consolidation: A neurobiological perspective. Current Opinion in Neurobiology, 5, 169-177. doi:10.1016/09594388(95)80023-9
Storm, B. C. (2011). The benefit of forgetting in thinking and remembering. Current Directions in Psychological Science, 20(5), 291-295.

Tulving, E. (1974). Cue-dependent forgetting. American Scientist, 62(1), 74-82.

Underwood, B. J. (1957). Interference and forgetting. Psychological Review, 64(1), 49-60. doi:http:// dx.doi.org/10.1037/h0044616

Unsworth, N., Brewer, G., \& Spiller, G. J. (2013). Focusing the search: Proactive and retroactive interference and the dynamics of free recall. Journal of Experimental Psychology: Learning, Memory, and Cognition, 1-15. doi:10.1037/ a0033743

Villareal, D. M., Do, V., Haddad, E., \& Derrick, B. E. (2002). NMDA receptor antagonists sustain LTP and spatial memory: Active processes mediate LTP decay. Nature Neuroscience, 5(1), 4852. doi:10.1038/nn776

Winocur, G., Becker, S., Luu, P., Rosenzweig, S., \& Wojtowicz, J. M. (2012). Adult hippocampal neurogenesis and memory interference. Behavioural Brain Research, 227(2), 464-469. doi:10.1016/j.bbr.2011.05.032

Wixted, J. T. (2004). The Psychology and Neuroscience of forgetting. Annual Review of Psychology, 55, 235-269. doi:10.1146/annurev. psych.55.090902.141555

Wixted, J. T. (2010). The role of retroactive interference and consolidation in everyday forgetting. In S. Della Sala (Ed.), Forgetting (pp. 285-312). New York: Psychology Press. doi:10.1037/ a0020874

Wixted, J. T., \& Rohrer, D. (1993). Proactive interference and the dynamics of free recall. Journal of Experimental Psychology: Learning, Memory, and Cognition, 19(5), 1024-1039. 